\section{The Volcanism of lo}

\author{
G. Israel, Verrières Le Buisson
}

(CNRS Service d'Aéronomie)
Through the observations of the planets from space, many natural phenomena, similar to those be found on Earth have been revealed. For example, we now have proof of an activity on Mars which gives rise to morning ice fogs and the occasional appearance of veritable tempests of dust in the rarefied atmosphere that surrounds the planet. We know also that the atmosphere of Venus is the scene of electrical storms, and reverberates to constant thunder and lightning. The great red spot on the disc of Jupiter can be interpreted as a gigantic atmospheric cyclone of tropical intensity. Other features of the Jovian image such as the ovals which accompany the red spot are equally of meteorological origin, similar in nature to those experienced with our own atmosphere.

Without question however, in this series of recent discoveries, the observations made by the camera probes of Voyager, of the surface of the satellite lo, have been the most astonishing. For the first time, it has been possible on the surface of another object in the solar system to record a pheno- menon which in spectacular fashion resembles a volcanic eruption.

Orbiting at a distance of $350200 \mathrm{~km}$ from the "surface" of the planet Jupiter, lo is the nearest of the four galilean satellites (see Table). In its mass and diameter it resembles the Moon, and like the Moon it cannot retain a gaseous envelope; atmospheric pressure is around $10^{-7} \mathrm{~atm}$. But unlike the Moon, lo cannot be regarded as dead, on account of the relative nearness of its grand possessor (Fig. 1). Its orbital radius is only six times the radius of Jupiter whilst the Moon orbits the Earth at a radius of 56 times the Earth's radius $(356410 \mathrm{~km}$ at the perigee). It is evident therefore that lo is immersed in the magnetic atmosphere of Jupiter while the Moon skims through only the tail of the Earth's magnetosphere (Fig. 2).

Even if the Moon-Earth distance is approximately the same as the lo-Jupiter distance, owing to the enormous mass of Jupiter, its satellite is subjected to tidal forces that are much greater than those that act on the Moon. Moreover, as lo is
Fig. 2 - Although similar to that of the Earth, the magnetosphere of Jupiter, under the influence of a dipole field 4000 times as strong as the Earth's, rotates with the planet. The concentration of charged particles is highest in the toroidal region created by 10.

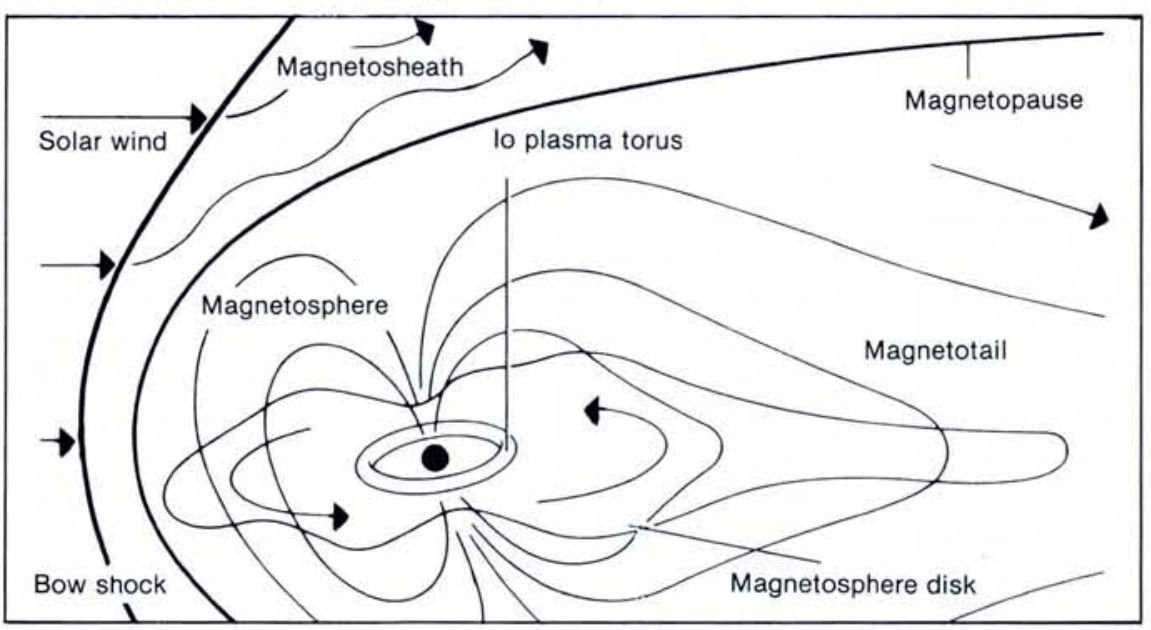

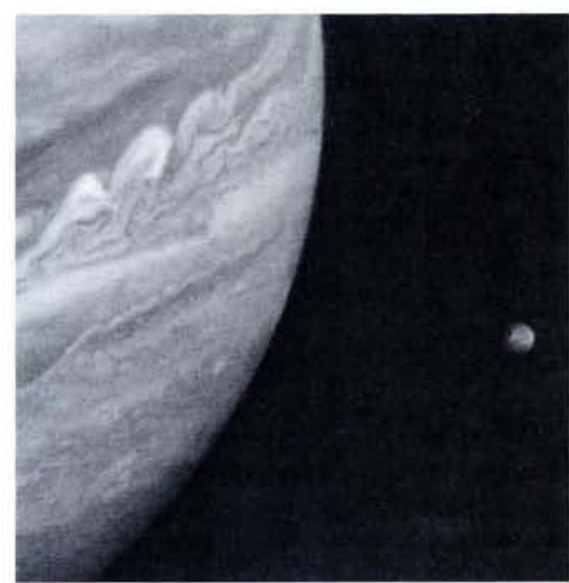

Fig. 1 - Jupiter and its satellite 10

rotating synchronously with its planet, its internal structure suffers a permanent deformation.

If lo maintained a constant distance from Jupiter that would be the sum of the consequences, but because of the proximity of the other Jovian satellites, notably Europa and Ganmymede, its orbit has been constantly perturbed resulting in an excentricity which is not negligible $(e=0.0043)$. On each revolution, the tidal forces on lo go through a maximum and minimum imposing on the internal layers, gravitational strains, the energy of which is dissipated in the form of heat. While the principal source of energy in the terrestrial planets and the Moon, is the disintegration of radioactive elements contained in the rocks such as thorium and potassium, in lo, the dominant contribution is from the release of gravitational strains.

Assuming lo to be like the Moon, a homogeneous body comprising a solid core covered by a mantle, the quantity of heat to be dissipated would be 10 times that of the Moon. Probably however, the core of lo has become liquid, as the core of the Moon is already close to a phase change, and this would result in a decrease in the thickness of the mantle and an acceleration of the dissipative phenomenon described above.

How then can the thermal energy escape? Calculations show that thermal conduction through solid material in a body the size of lo cannot be the explanation and it is greatly to the credit of Peale, Cassen and Reynolds that they predicted heat

\begin{tabular}{|c|c|c|c|c|c|c|c|}
\hline & $\begin{array}{l}\text { Sidereal } \\
\text { Period } \\
\text { (Day) }\end{array}$ & $\begin{array}{l}\text { Distance from } \\
\text { the Planet Surface } \\
(\mathbf{k m})\end{array}$ & $\begin{array}{l}\text { Distance to } \\
\text { the Centre of } \\
\text { the Planet }{ }^{1}\left(R_{\mathrm{J}}\right)\end{array}$ & $\begin{array}{c}\text { Diameter } \\
(\mathbf{k m})\end{array}$ & $\begin{array}{l}\text { Density } \\
\left.\text { (g/ } \mathrm{cm}^{3}\right)\end{array}$ & $\begin{array}{c}\text { Albedo }^{2} \\
(\%)\end{array}$ & $\begin{array}{c}\text { Nature } \\
\text { of the Surface }\end{array}$ \\
\hline 10 .......... & 1,769 & 350200 & 5,90 & 3690 & 3,53 & 63 & $\begin{array}{l}\text { particles \& sulphur } \\
\text { and frozen sulphurated } \\
\text { substances }\end{array}$ \\
\hline Europa. & 3,559 & 599500 & 9,40 & 3130 & 3,03 & 46 & ices \\
\hline Ganymede. . . & 7,155 & 998600 & 14,99 & 5280 & 1,93 & 43 & ices \\
\hline Callisto ... . . . . & 16,689 & 1808600 & 26,33 & 4840 & 1,79 & 17 & ices and rocks \\
\hline
\end{tabular}

1. One Jorian radius $\left(R_{\jmath}\right)$ equals $71398 \mathrm{~km}$.

2. The albedo of the Moon is $12 \%$. 


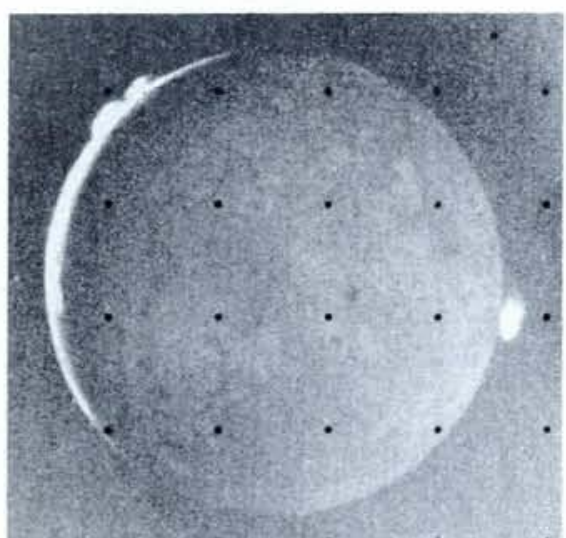

Fig. 3 - Plumes from three volcanoes photographed by Voyager 2.

dissipation through volcanic activity with large gaseous emissions, several months before the confirmation came from the Voyager missions.

Fig. 3 shows one of the photographs taken - at a distance of $10^{6} \mathrm{~km}-$ by Voyager 2 under the most advantageous lighting conditions. Three eruptions can be seen, two on the crescent side and one on the darkened edge. The last of these (Loki) observed four months previously by Voyager 1 , is the largest so far measured -32 $\mathrm{km}$ across and $185 \mathrm{~km}$ high.

Deprived of any dense atmosphere that could generate clouds or hold up aerosol layers (unlike Titan the satellite of Saturn which is obscured by a veil of aerosols) lo has a surface that is directly observable in the visible spectrum. The highly reflective disc of lo ( $60 \%$ reflectivity for sun-light) has a yellow orange appearance and this, strongly supported by UV and IR spectroscopic measurements, indicates that sulphur is responsible.
Comparing two photographs taken at six-hour intervals, leads to the conclusion that gas is ejected in great puffs, and the most reasonable explanation for certain blotches that can be seen on pictures taken by the Voyager cameras, is that in the eruptions, sulphur dioxide gas is emitted which condenses at high altitude, falls to the ground and there forms ice deposits that have the characteristic blue colour observed. In addition, spectroscopic analysis of the chemical composition of the tenuous atmosphere of lo confirms that $\mathrm{SO}_{2}$ is the principal component.

That is not all, as one of the most surprising features of Jupiter's atmosphere is related. The planet is surrounded by a plasma ring, the major radius of which corresponds to the orbital radius of lo and the minor radius to the planet's radius. Among the charged particles which make up this torus-shaped plasma, ions of sulphur and oxygen have been clearly identified, and in view of the geometric configuration of the planet and its satellite, the source can only be lo. The particles are very strongly ionized and UV spectrometry from the Voyager probes has shown up very clearly the $\mathrm{S}^{++}$ ion which emits at $690 \AA$

This does not mean to say that we understand entirely the physics of the eruption phenomenon. Analogy with the Earth is not very satisfactory as volcanic gases on Earth are predominantly water vapour and $\mathrm{CO}_{2}$. How then interpret the selective resorption of sulphur which seems to have determined the formation of lo? What is certain at least is that a massive restructuring of the surface is going on even today, the proof of which lies in the almost total absence of impact craters on the surface as seen from high precision photographs (with a resolution less than $1 \mathrm{~km}$ ). This is all the more remarkable as the neighbouring Jupiter will have served as a major centre of gravitational attraction for the myriad objects of varying size that have peppered the Jovian space since earliest times.

Looking at the situation from a geological point of view, it is possible to explain the topography of 10 , in particular that of the zones corresponding to the eight giant eruptions identified in the course of the Voyager missions. From four images taken by Voyager 1 on 4 March 1979 at a distance of $377000 \mathrm{~km}$, the engineers at NASA have constructed the picture shown in Fig. 4. The heart-shaped patch $(700 \times 1000$ $\mathrm{km})$ to be seen left centre, corresponds to the eruption of the volcano Pele; above and to the left can be seen the volcano Loki, the spectacular plume of which was photographed by Voyager 2 (Fig. 3). The circular form of the contours must be the result of fall out of the material making up the plume.

The volcanic activity of lo shows itself in another form. On Fig. 5, taken with a high resolution camera by Voyager 1 on 5 March 1979 , a crater of $50 \mathrm{~km}$ diameter can be seen that is analagous to terrestrial craters with characteristic lava runs down the sides, surrounding a central bowl.

In the case of lo, detailed analysis suggests that the runs are of a material of low viscosity - perhaps molten sulphur. Curiously, the regions where traces of old volcanoes can be seen do not correspond to those of high relief: in the equatorial regions where most of the volcanoes are situated, there are no hills or mounds higher than $1 \mathrm{~km}$. (Volcanoes with a similar
Fig. 4 - Mosaic of four images transmitted by Voyager 1 which has enabled the surface relief to be studied in the regions of the eruptions.

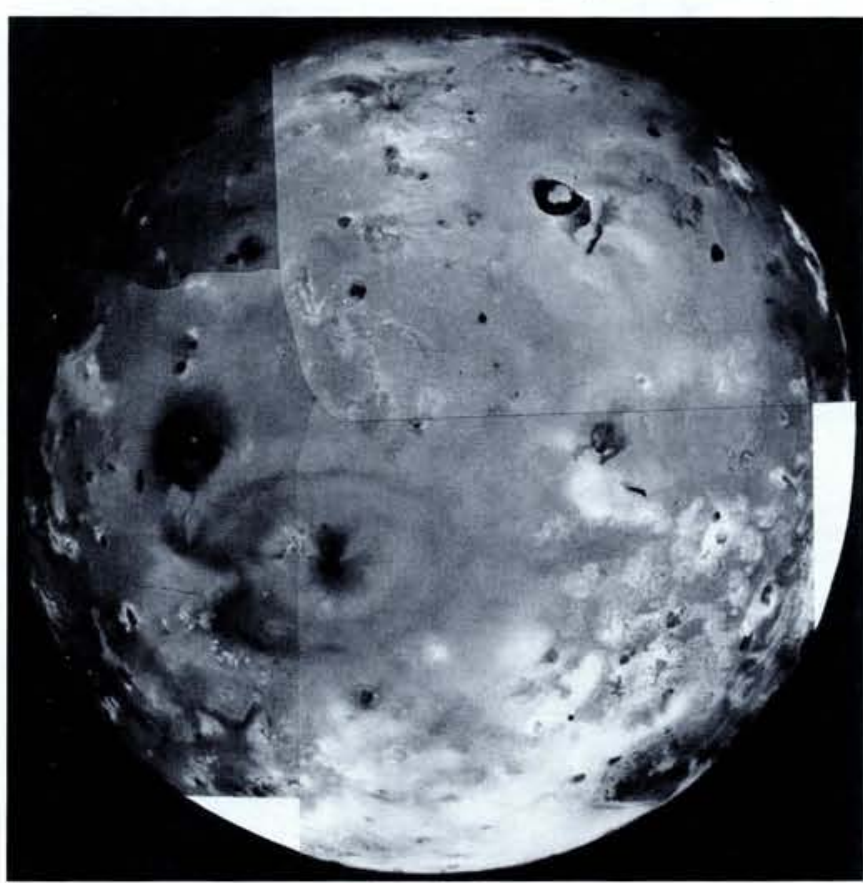

Fig. 5 - Image of a crater on the surface of 10 , recorded by Voyager 1 with a resolution of $0.3 \mathrm{~km}$, showing the characteristic liquid runs.

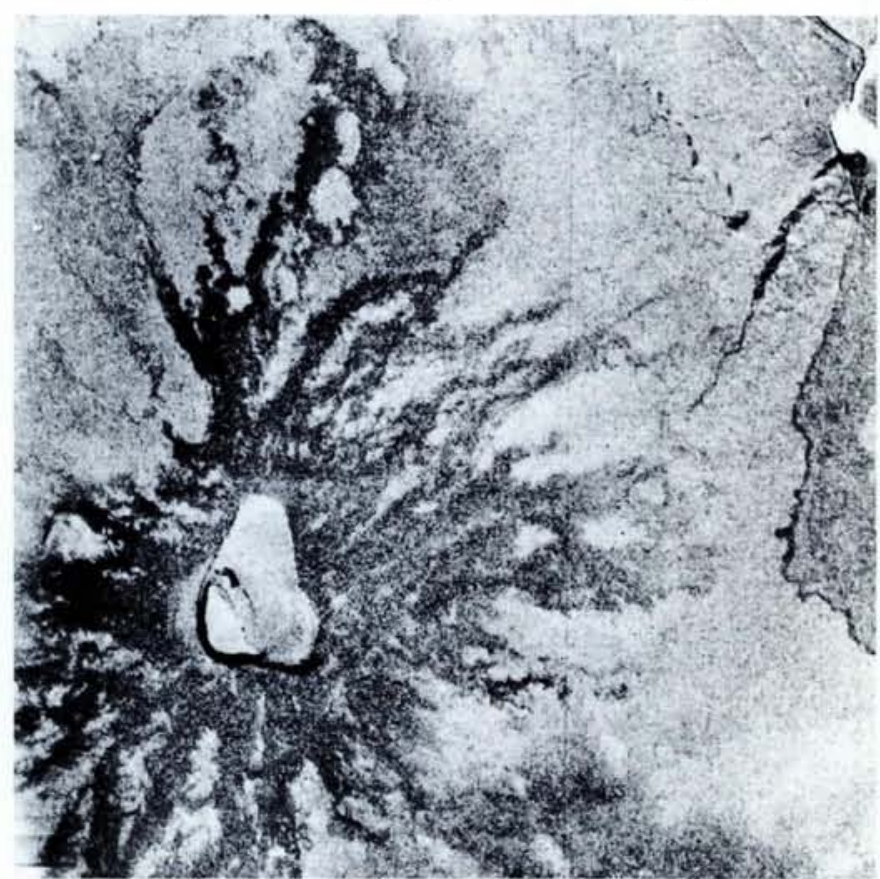


morphology are also to be found on the Earth's surface in New Mexico.) Nearer the poles of lo, the terrain is more irregular and several mountain ranges are visible with peaks several kilometres high.

Shortly after the two fly-bys of lo, geochemists set out to describe the composition of the satellite's crust. Any interpretation of the eruption processes has to be compatible with the temperatures registered by the probes, which indicated that the surface was in the main very cold $\left(-146^{\circ} \mathrm{C}\right)$. On the other hand, zones of higher temperature have been located, notably in the vicinity of the volcano Loki where temperatures in excess of $17^{\circ} \mathrm{C}$ have been measured. This is nevertheless much below the melting point of sulphur $\left(112^{\circ} \mathrm{C}\right)$ and the theory has been advanced that a solid layer of sulphur and $\mathrm{SO}_{2}$ is covering an ocean of liquid sulphur (Fig. 6 ). In the model of B. Smith (University of Arizona), the plumes would be produced by volcanic eruptions of a phreatic type, such as is observed on Earth.

Other models have also been suggested but many questions remain, notably on the interface between lo and the Jovian magnetosphere. Even if lo is the source of the plasma ring, the mechanism of its formation is far from clear. How do the minute dust particles which make up the plume succeed in escaping from lo's atmosphere? In order to overcome lo's gravity, neutral particles would have to travel much faster than observed. In addition, there are certain indications that ions constituting the plasma are formed in the middle of the

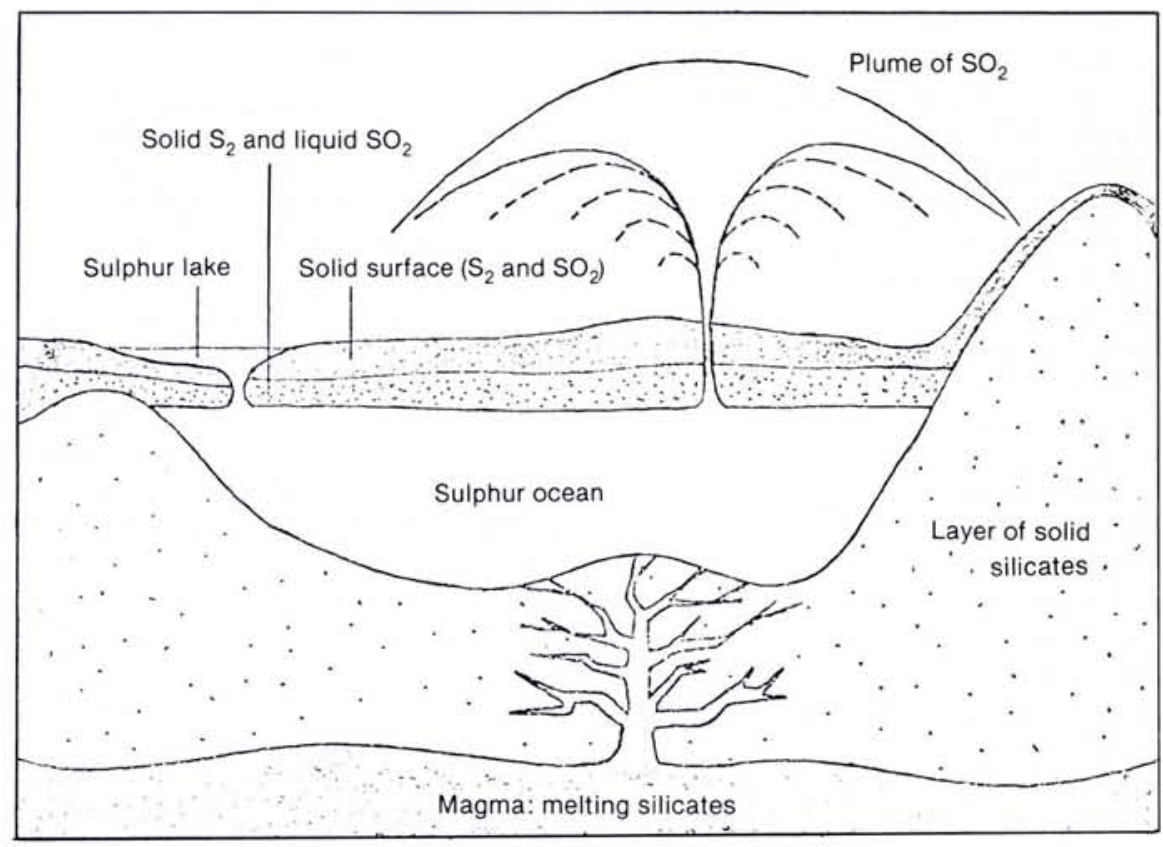

Fig. 6 - Model of the crust of lo (after B. Smith)

torus space, in which case by what process are dust particles dispersed into the torus?

Torrance Johnson, of the Jet Propulsion Laboratories, has suggested that magnetospheric electrons, bombarding minute dust particles in the volcanic plumes, could charge them sufficiently to be carried off by the sweeping Jupiter magnetic field. Once free, dust particles could break up into atoms and molecules, and become ionized. Alternatively, the plumes of lo might not be the only source of ions and a sputtering mechanism acting on the surface material of lo could be contributing; the charged particles in the magnetosphere would be rather effective in liberating atoms of sulphur and oxygen as well as sodium. This process was in fact first put forward to ex plain a phenomenon observed from the Earth, namely the formation of a narrow belt of neutral sodium atoms round Jupiter in the plane of the orbit of lo. Moreover, recently it has been observed that jets of matter are ejected intermittently from the disc of lo labelled with the spectroscopic line at $5890 \AA$ that characterises sodium.

\section{Problems of Physics in the Developing Countries of Europe}

In the week before the General Conference, the Physics and Society Committee held a seminar at the Bogaziçi University aimed at exploring the problems of physicists in the less endowed areas of Europe. As the seminar was also sponsored by UNESCO, "developing" was the term employed and meant those nations that the UN indicator showed to have a standard of living below or close to a minimum level. However, it became evident that although there was a general correlation between physics activity and GNP/capita or similar indices, the parallel was by no means exact. Detailed reports were presented by a number of delegates on the state of physics in their country and whilst a low level of funding and the absence of any science policy were common complaints, others were specific to the particular region.

\section{Portugal}

Portugal's problems begin at the level of secondary education, where there has been an explosion in the school population so that experimental studies and physics in particular have suffered disproportionately. Moreover, not only have laboratory facilities always been inadequate, but physics forms simply part of a general science course, then is linked to chemistry. Only in the final year is it separate subject.

A trend towards a similar system can be observed in a number of advanced nations and these would do well to note the effects in Portugal. Few of the teachers have a physics background and so students do not feel motivated towards the subject. As a result few choose physics and, as university students are required to define their subject and are then selected by the Education Ministry on the basis of their past results, many of the students in physics are rejects from other subjects. The problem has been further aggravated by the fact that only since 1964 have degrees been awarded in physics and now, as the quality at the top tends to rise, the effect on teaching is adverse, because of the call of research. Research is restricted by inadequate buildings, equipment and adminis- trative infrastructure, the difficulties of access to important experimental equipment and the sense of isolation that results. International cooperation does exist but almost none with the immediate neighbour Spain and participation in any international meeting is difficult because of lack of funds.

\section{Spain}

Spain with a total investment in research and development of $0.3 \%$ of its GNP is only marginally ahead of Portugal and physics would seem to have been relatively badly treated in comparison with other scientific subjects because, it was suggested, of the absence of any "friends at court" over too many years. Out of the total number of active physicists, estimated to correspond to 26 / million of the population, two thirds are concentrated in Madrid, which implies a serious starvation in the provincial universities. Solid state physics is the dominant subject which corresponds neither to the social needs of the country where perhaps the earth sciences 